\title{
Quantum Current in Graphene Nano Scrolls Based Transistor
}

\author{
Sayed Norollah Hedayat ${ }^{1, *}$, Mohammad Taghi Ahmadi ${ }^{1}$, Hassan Sedghi ${ }^{1}$, \\ Hadi Goudarzi ${ }^{1}$ \\ ${ }^{1}$ Department of Physics, Faculty of Science, Urmia University, Urmia, Iran
}

\begin{abstract}
Graphene based material application as a new centuery material are growing rappidly its carrier transport phenomenon with fast mobility have been focused resently. In the graphene family nanoscrolls because of their especial structure need to be explored. In the presented work a theoretical model for carrier transport in the arcemedus graphene nanoscrolls is reported. Graphene nanoscroll chairal dependent electrical property is considered and then schottky transistor based platform is modeled. The transport coeficient as a fundamental transport factor is discussed. The geometrical paprameter effect on the working phenomenon is considered as well.
\end{abstract}

Keywords: quantum current, degenerate and nondegenerate approximation, graphene Nano scrolls, transmission coefficient, transistor.

Date of Submission: 28 January $2017 \quad$ Date of Accepted: 17 March 2017

\section{INTRODUCTION}

Graphene Nano scroll (GNS) as a rolled up structure of graphene because of its scroll shape cross section totally is different from carbon nanotubes. It has been found that the scrolled configurations of graphene indicate higher stability in terms of their structure, as opposed to their counterpart planar-configuration carbon nanotubes. The energy dispersion relation of graphene Nano scrolls has been determined using the tight-binding (TB) model. It has been reported that the electronic structures of Nano scrolls are significantly dependent on the chirality number $(\mathrm{n}, \mathrm{m})$. Armchair Nano scrolls are metallic or semi-metallic depending on their spiral length, and metallic Nano scrolls have a large energy gap at the Fermi level [3-4]. Zigzag Nanoscrolls are small bandgap semiconductors when $(n-1)$ is a multiple of 3; otherwise, they are semiconductors with an observable energy gap at the Fermi point. The resulting high-quality graphene Nano scrolls show promising applications in a wide variety of electronic devices. Recently graphite based materials such as grapheme nanoribbon has been studied extensively [1-2]. And in all nanotechnology related fields including transistor fabrication interconnecting circuitry, electromechanical switches infrared emitters and bio- sensors have been employed widely [5-6]. Even long mean free path and also electron-electron interactions with high level of electron scattering exist in graphene [12-13]. By considering all these unique properties of carbon nanostructures specially GNSs with distinguished properties it is completely accepted as a promising component in future electronic technology [14]. In the presented work as shown in Figure (1) and Figure (2) a GNS transistor with two schottcky contacts is considered which can be made by top-down fabrication method.

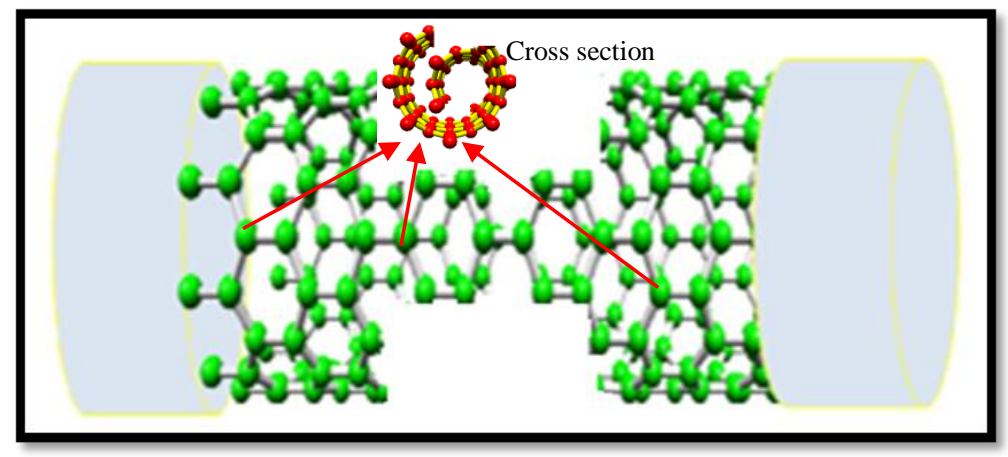

Figure (1). Schematic of two schottcky contacts of metallic GNS with semiconducting in the channel region. Also a simple fabrication method to GNS production has been proposed by wet chemistry technique [7-8] as well as hydrogenation on one side that can be scrolls up it completely into a graphene Nanoscroll (GNS) which is stable over the room temperature [9-10]. Since a GNS can be considered as a rolled up graphene sheet into a 
spiral form, band structure of graphene forms the basis of all types of GNSs and so the two dimensional dispersion relation of grapheme can be determined using the tight-binding approximation (TB) as.

$$
E(k)= \pm t \sqrt{1+4 \cos \left(\frac{3 k_{x} a_{c c}}{2}\right) \cos \left(\frac{\sqrt{3} k_{y} a_{c c}}{2}\right)+4 \cos ^{2}\left(\frac{\sqrt{3} k_{y} a_{c c}}{2}\right)}
$$

Where the positive and negative signs indicate conduction and valence bands respectively, $\mathrm{t}=3 \mathrm{eV}$ is the nearestneighbor C-C bonding energy, $\mathrm{k}$ denotes on wave vector $(k=k x \hat{\imath}+k \hat{j})$ and $a_{c-c}=1.42 A^{\circ}$ is the nearest neighbor $\mathrm{C}-\mathrm{C}$ bonding distance.

For semiconductor type, the edge track of the GNS is around (for example y-axis), consequently the boundary condition of $\vec{k} . \vec{c}$ is imposed around the y-axis. Because the phase transfer of $k$ thus $\vec{k} \cdot \vec{c}=2 \pi j-\theta$, where $j=1,2, \ldots, n$ for chirality $(n, 0)$ at the same time as for periodic boundary condition in the region of the $y$ axis is equated and $n k_{y, j} a=2 \pi j-\theta, k_{y, j} a=\frac{2 \pi j-\theta}{n}$ are considered:

$$
E^{ \pm}(k)= \pm t\left[1+4 \cos \left(\frac{\sqrt{3} k_{z} a}{2}\right) \cos \left(\frac{j \pi}{n}-\frac{\theta}{2 n}\right)+4 \cos ^{2}\left(\frac{j \pi}{n}-\frac{\theta}{2 n}\right)\right]^{1 / 2}
$$

where $\mathrm{k}_{\mathrm{z}}$ is a quantize vector that is based on the Schrodinger's equation and $\mathrm{n}$ is the number of the sub-bands where $\mathrm{n}=1,2, \ldots \mathrm{n}$. Parameter $a$ is the distance between the closest carbon to carbon atom which is equal to $1.42 \AA$, according to Chen et al. The value of $t$ is set to $2.5 \mathrm{eV}$.

\section{Model}

As shown in Figure (2) the schottcky structure in the presented model is employed in regions 1and 3 the potential energy is estimated to be zero, and in region 2 the potential energy is $V_{0}$ therefore[11],

$$
k_{1}=k_{3}=\sqrt{\frac{2 m E}{\hbar^{2}}}, k_{2}=\sqrt{\left(E-E_{g}\right) \frac{2}{3 t a_{c c}}}
$$

The transmission ratio differs in terms of probability of current density according to $\mathrm{T}$ equation

$$
T=\left|\frac{j_{\text {transmitted }}}{j_{\text {incident }}}\right|=\left|\frac{A_{n+1}}{A_{n}}\right|^{2}
$$

The modified one dimensional dispersion relation for the further development is considered and the transmission amplitudes for a single square barrier centered at the origin with height $V_{0}$ and width bas shown in the Figure (2) is analytically modeled.

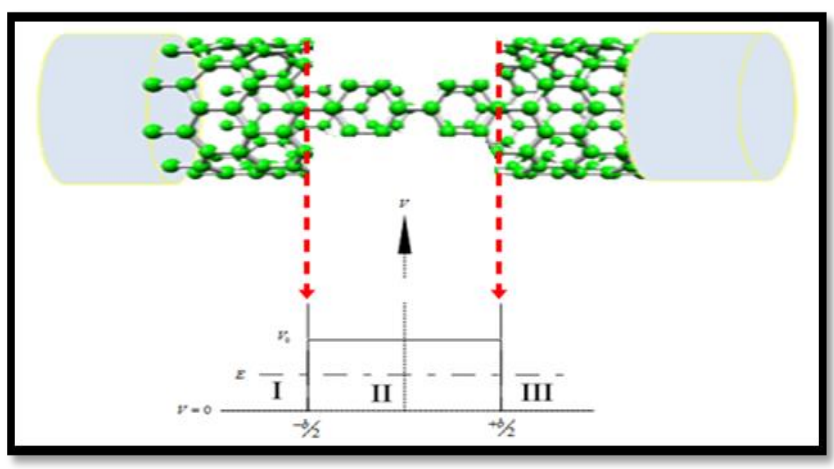

Figure (2). Channel region barrier in GNS based schottky contact.

The transistor operation can be modeled in the form of three different regions as shown in Figure (2), which leads to the Schrödinger equation result for potential of each region thus the transmission coefficient has been explained by transmission amplitude, concentration and total power of a transmitted wave. The transmission 
coefficient is a scale of how an electromagnetic wave (light) propagates throughout a material which is the transmission amplitude to power of two therefore the transmission coefficient $\mathrm{T}$ can be calculated as:

$$
T=|t|^{2}=\frac{4 \frac{K^{2}}{k^{2}}}{-1+2 \frac{K^{2}}{k^{2}}-\frac{K^{4}}{k^{4}}+\left(1+2 \frac{K^{2}}{k^{2}}+\frac{K^{4}}{k^{4}}\right) \cosh ^{2}(b K)}
$$

Where $\mathrm{b}$ is the length of the barrier, the wave vector $k$ outside the barrier is a real quantity for all positive energies $E$ of the electron. The conduction band minimum in the region outside the barrier is taken as the zero of energy. Since $k$ and $K$ are both dependent on effective mass and energy, hence for different material pairs the variation of the transmission coefficient for energy values with respect to the barrier height will be different [15-16-17]. For $\left(E_{n}=\frac{E}{V_{0}}\langle 1)\right.$, the transmission coefficient increases from 0 to 1 in a non-linear fashion by increasing the wave number as shown in Figure (3) for different regions.
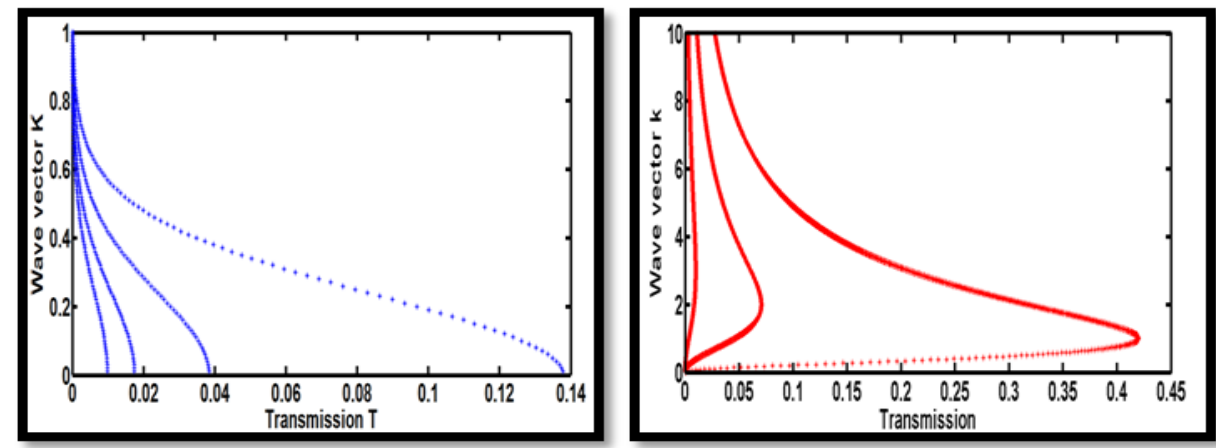

Figure (3). Transmission for different wave vector values in the GNS at channel region.

As shown in Figure (3), by changing the energy in allowed range the transmission value also is changed. if the energy of electron in each region is changed. On the other hand, the geometrical parameter effect on the transport factor is investigated as shown in Figure (4).
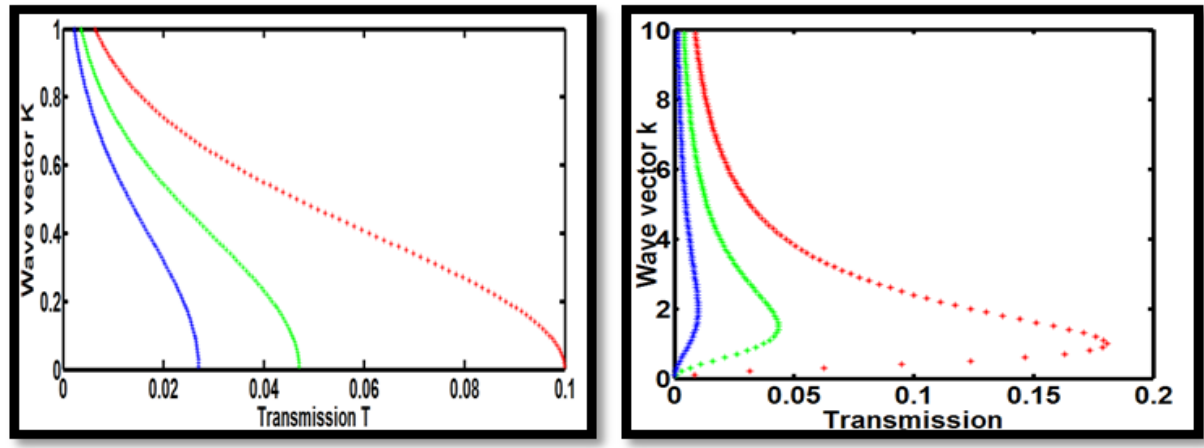

Figure (4). the transmission variation based on the channel region length (red line indicates channel length 3 $\mathrm{nm}$, green line for channel length $5 \mathrm{~nm}$, blue line for channel length $10 \mathrm{~nm}$ ).

In the presence of $k^{2}$ and $K^{2}$ the transmission is modified as:

$$
T(E)=\frac{4 \alpha\left(1-\frac{E_{g}}{E}\right)}{-1+2 \alpha\left(1-\frac{E_{g}}{E}\right)-\alpha^{2}\left(1-\frac{E_{g}}{E}\right)^{2}+\left(1+2 \alpha\left(1-\frac{E_{g}}{E}\right)+\alpha^{2}\left(1-\frac{E_{g}}{E}\right)^{2}\right) \cosh ^{2}\left(b \sqrt{\beta\left(E-E_{g}\right)}\right)}
$$

As shown in Figure (5), the transmission coefficient as a function of barrier height is numerically simulated and it is concluded that the optimum energy in each system is closer to the barrier height. 


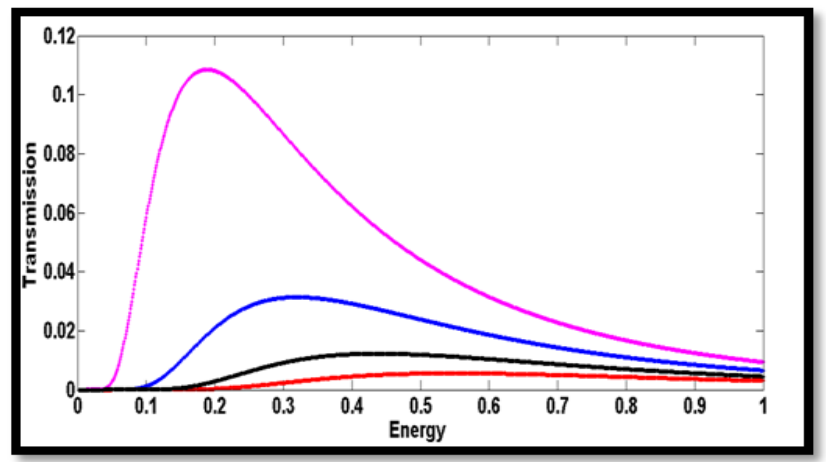

Figure (5). the transmission variation for energies less than barrier high.

it is observed that in the energies more than barrier height the transmission saturates at $\mathrm{E}>\mathrm{V} 0$ as shown in Figure (6).

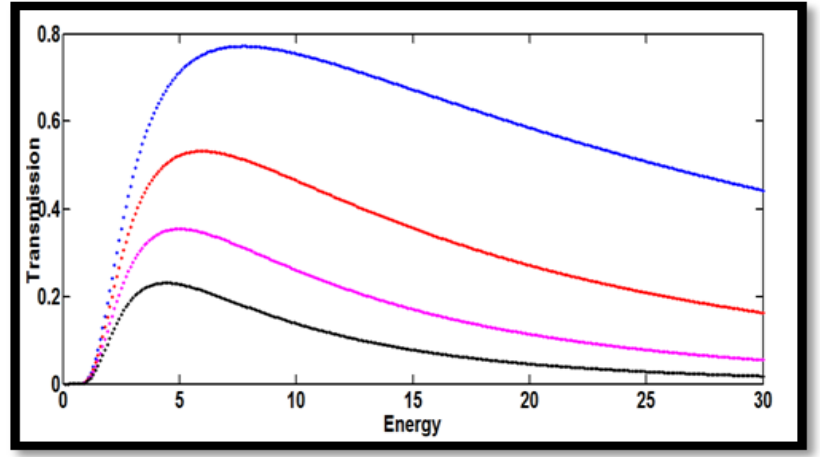

Figure (6). The T-E characteristic with channel length variation for energies more than barrier height ( $b=0.5$ (black), $b=0.4$ (violet), $b=0.3$ (red), $b=0.2$ (blue) ).

Also the quantum current in the formalism of Land Auer formalism is developed and numerically illustrated as shown in Figure (7).

$$
I=\int_{0}^{\eta} \frac{4 \alpha K_{B} T\left(1-\frac{E_{g}}{E}\right)}{-1+2 \alpha\left(1-\frac{E_{g}}{E}\right)-\alpha^{2}\left(1-\frac{E_{g}}{E}\right)^{2}+\left(1+2 \alpha\left(1-\frac{E_{g}}{E}\right)+\alpha^{2}\left(1-\frac{E_{g}}{E}\right)^{2}\right) \cosh ^{2}\left(b \sqrt{\beta\left(E-E_{g}\right)}\right)} \frac{1}{1+\exp (x-\eta)} d x
$$

Where $\frac{E-E_{g}}{k_{B} T}=x, \frac{E_{g}-E_{f}}{k_{B} T}=-\eta, \alpha=\frac{\hbar^{2}}{3 t m a_{c c}}, \beta=\frac{2}{3 t a_{c c}}, K_{B}$ is Boltzmann's constant and T is temperature.

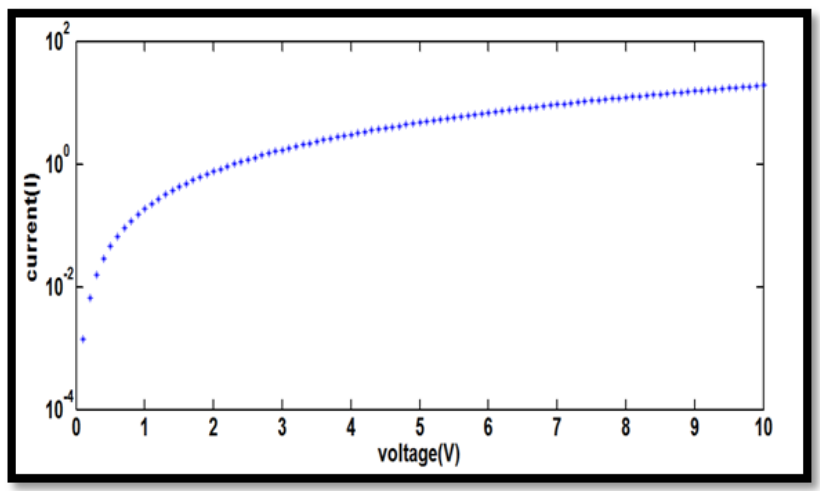

Figure (7). Current -voltage characteristic of GNS transistor based on the presented model.

Numerical investigation on I-V characteristic indicates the Ohmic response on low biases on the other hand the current is saturated for high applied voltages. 


\section{CONCLUSION}

Graphene nanoscroll a tubular carbon based material with chirality dependent band gap rarely has been explored. It's simple fabrication method together with notable electrical property helps to progress GNS based electronic. GNS based device exploration start with understanding of transport phenomenon on this structure therefore in the presented work carrier transport model for GNS based schottky contact theoretically is explored and numerical simulation on the proposed model is illustrated. Similar to the conventional schottky contacts the Ohmic and saturated current voltage characteristic is detected. Additionally, structure dependence characteristic is reported.

\section{REFERENCES}

[1]. Y. G. Gogotsi, et al., "Materials: Transformation of diamond to graphite," Nature vol. 401, pp. 663-664, 1999.

[2]. T. Irifune, et al., "Materials: Ultra hard polycrystalline diamond from graphite," Nature, vol. 421, pp. 599-600, 2003

[3]. Zeng, F., et al., Facile Preparation of High - Quality Graphene Scrolls from Graphite Oxide by a Micro explosion Method. Advanced Materials, 2011. 23(42): p. 4929-4932.

[4]. Martins, B.V.C.andD.S.Galvao, Curved graphene nanoribbons: structure and dynamics of carbon Nano belts. Nanotechnology, 2010. 21(7).

[5]. T. O. Wheeling, et al.,Molecular Doping of Graphene," Science Nano Lett., vol. 8, pp. 173-177, 2004.

[6]. D. J. K. S. Novoselov, F. Schedin, T. J. Booth, V. V. Khotkevich, S. V. Morozov and A. K. Geim, "Two-dimensional atomic crystals," Proc. Natl Acad. Sci, vol. 102, pp. 10451-10453, 2005.

[7]. Xie, X., et al., Controlled Fabrication of High - Quality Carbon Nanoscrolls from Monolayer Graphene. Nano Letters, 2009. 9(7): p. $2565-2570$.

[8]. Lima, M.D., et al., Biscrolling Nanotube Sheets and Functional Guests into Yarns. Science, 2011. 331(6013): p. 51- 55.

[9]. Zhu, S.and T. Li, Hydrogenation enabled scrolling of graphene. Journal of Physics D - Applied Physics, 2013. 46(7).

[10]. Shi, X.H., et al., A translational Nano actuator based on carbon Nano scrolls on substrates. Applied Physics Letters, 2010. 96(5).

[11]. S.N.hedayat and M.T.ahmadi, et al., Quantum Current Modelling on Graphene Nano scrolls. University of Diyala Dec 2015, pp599-605.

[12]. F. Guinea, et al., "Electronic properties of stacks of graphene layers," Solid State Communications, vol. 143, pp. 116-122, 2007.

[13]. H. Li, et al., "Carbon Nanomaterials for Next-Generation Interconnects and Passives: Physics, Status, and Prospects," IEEE Transactions On Electron Devices, vol. 56, pp. 1799-1821, 2009.

[14]. M. Lundstrom and J. Gao, "Nanoscale transistors: device physics, modeling and simulation," Springer, Birkhäuser- Technology \& Engineering - 217 pages, 2006.

[15]. Physics of Quantum Well Devices, B.R.Nag, Klewer Academic Publishers.

[16]. Martin David Treharne Evans, Quantum Mechanical Tunnelling, July (2012).

[17]. Xi.Chen and Chun-Fang Li, Eur. Phys. J. B. 46, 433 (2005). 